\title{
A New Species of Neotropical Carpenter Ant in the Genus Camponotus (Hymenoptera: Formicidae), Apparently without Major Workers
}

\author{
William Mackay ${ }^{1}$ and Paola A. Barriga ${ }^{2}$ \\ ${ }^{1}$ Department of Biological Sciences, The University of Texas, El Paso, TX 79968, USA \\ ${ }^{2}$ Department of Biological Sciences, University of Arkansas, Fayetteville, AR 72701, USA
}

Correspondence should be addressed to William Mackay, wmackay@utep.edu

Received 19 April 2012; Revised 4 June 2012; Accepted 11 June 2012

Academic Editor: Martin H. Villet

Copyright (C) 2012 W. Mackay and P. A. Barriga. This is an open access article distributed under the Creative Commons Attribution License, which permits unrestricted use, distribution, and reproduction in any medium, provided the original work is properly cited.

\begin{abstract}
We describe a new species of carpenter ants from Ecuador, which apparently has an obligatory relationship with the ant plants Cecropia membranacea Trécul, C. herthae Diels and C. marginalis Cuatrec. The workers are relatively small and hairy, and based on a number of collections, it does not appear to have major workers. We compare the new species to Camponotus balzani, to which it appears to be similar and which has normal major workers, and also lives in Cecropia spp.
\end{abstract}

\section{Introduction}

Camponotus carpenter ants have a worldwide distribution and form one of the hyperdiverse genera together with Pheidole [1]. It is still unknown which of these two largest genera has the greater number of species as many new species await discovery. In the New World, about 650 taxa have been named in Camponotus, which will be reduced to about 440 valid species, including about 140 new species (Mackay, unpublished, manuscript available at http://www.utep.edu/leb/antgenera.htm). Other species of neotropical Camponotus have been recently described [2-4].

The revision of such a large group has taken nearly 20 years, with several more needed to complete the work. Occasionally names are needed for ecological studies, which cannot wait the completion of the entire work. We here describe an interesting new species to provide a name for an ant whose interaction with Cecropia will be described by the junior author in future papers.

This species is unusual in that the workers appear to be monomorphic. The majority of the carpenter ants are dimorphic, but monomorphic workers are also found in the montivagus species complex [5,6], subgenus Dendromyrmex [7], and some of the species in the subgenus Myrmobrachys are only weakly dimorphic.

Placement of this species into a subgenus is difficult because of the lack of major workers. Based on its similarity to C. balzani and several characters listed below, we placed it in the subgenus Tanaemyrmex. The clypeal carinae of the minor worker, female, and to some extent the male are well developed. The sides of the clypeus diverge anteriorly in all three castes. The head of the minor worker is longer than broad, with large eyes that reach the sides of the head. The minor worker is relatively large, with an elongated mesosoma, which is typical for the subgenus Tanaemyrmex. The dorsum of the mesosoma of the minor is convex and rounded and somewhat angulate between the two faces. The propodeal spiracle is elongated. The petiole is somewhat thickened (viewed from side). Appressed pubescence is sparse. The head and mesosoma are coriaceous, and the gaster is finely striolate. These characteristics are similar to most other species considered to be in Tanaemyrmex. 


\section{Materials and Methods (Measurements and Indices)}

At least ten individuals of each of eight species of Cecropia were found that were at least $100 \mathrm{~m}$ apart. The exceptions were C. litoralis Snethl, in which only two individuals were found and $C$. marginalis where eight plant individuals were collected. Camponotus reburrus was found in saplings of Cecropia membranacea and C. marginalis, but the majority of samples came from $C$. herthae. Seventy-five Cecropia individuals were collected, and C. reburrus was found in $9.3 \%$ of the trees collected.

Specimens were measured using an ocular micrometer in a dissecting microscope. The following abbreviations are used (all measurements in $\mathrm{mm}$ ):

HL: head length, anterior of median lobe of clypeus to mid-point of posterior margin;

HW: head width, maximum excluding eyes;

SL: scape length, excluding basal condyle;

EL: eye length, maximum dimension;

CL: clypeal length, from tops of posterior lobes of clypeus to anterior median edge;

CW: clypeal width, measured at level of tentorial pits;

WL: weber's length, anterior border of pronotum to posterior border of lobe of metapleural gland;

FFL: front femur length (maximum);

FFW: front femur width (maximum);

CI: cephalic index, $\mathrm{HW} / \mathrm{HL} \times 100$;

SI: scape index, SL/HL $\times 100$ (note HL used instead of HW);

CLI: clypeal index, CLI, CW/CL × 100;

FFI: front femur index, FFW/FFL $\times 100$.

\section{Results}

The new species is characterized as follows.

Camponotus (Tanaemyrmex) reburrus Mackay n. sp. (Figures 1, 2, 3, 4, 5, 6, 7, 8, and 9).

Diagnosis. The minor worker of $C$. reburrus is a relatively small (4.4-6.6 $\mathrm{mm}$ total length) yellowish brown specimen, with a transversely striped yellow and brown gaster, or at least with yellow lateral splotches. The sides of the head are straight and parallel, and the carina on the clypeus is well marked. The antennal scape has numerous erect hairs along the shaft, the hairs on the tibiae are coarse and suberect. Most surfaces are moderately to strongly shining.

The female of $C$. reburrus is a small (total length 6-7 $\mathrm{mm}$ ) hairy specimen, which is shiny dark brown with lighter colored legs. The gaster has lateral yellow splotches. The sides of the head are straight and parallel. The size and abundant

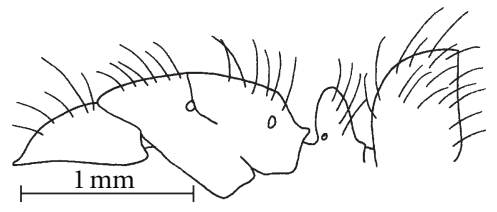

Figure 1: Mesosoma, petiole, and first gastral tergum of a minor worker of $C$. reburrus (Napo, Ecuador).

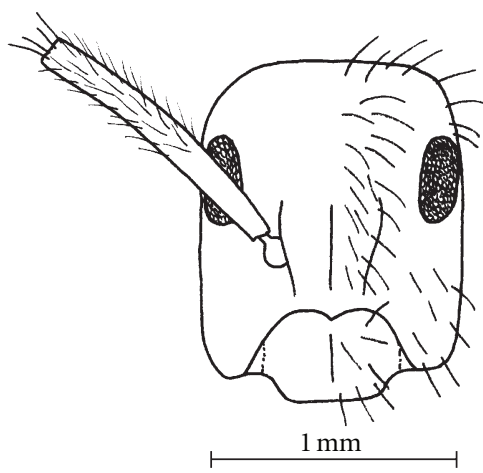

Figure 2: Head of a minor worker of C. reburrus (Napo, Ecuador).

erect hairs on the head and scape should separate it from all others in the subgenus Tanaemyrmex.

The male of $C$. reburrus is a small (total length $4.0-5.5 \mathrm{~mm}$ ) dark brown to yellowish brown specimen, with abundant hairs on most surfaces. Other than color and hairiness, it does not seem to possess characteristics which would distinguish it from other small males of Camponotus.

The major worker apparently does not exist.

3.1. Description of the Minor Worker. Minor worker measurements (mm, $n=4)$ : HL 1.20-1.38, HW 0.90-1.00, SL 1.061.14, EL 0.31-0.34, CL 0.36-0.45, CW 0.49-0.53, WL 1.641.84, FFL 0.96-1.08, FFW 0.30-0.31. Indices: CI 72-75, SI 8388, CLI 108-145, FFI 29-31.

Mandibles with 6 teeth; anterior border of clypeus nearly straight, carina well marked; sides of head straight, parallel, posterior border convex; eyes extend slightly past sides of head; scape extends nearly $1 / 2$ length past posterior lateral corner of head; mesosoma arched throughout, weakly depressed at sutures; dorsopropodeum about twice length of posteropropodeum, face weakly concave, spiracle circular; petiole moderately thickened when viewed in profile, apex rounded when viewed from front.

Erect hairs present on head, including surface of clypeus, cheeks, entire length of sides of head, posterior border, between frontal carinae, frons, ventral surface of head, scape with short $(0.05 \mathrm{~mm})$ erect and suberect hairs, erect hairs abundant on mesosoma, petiole and gaster, hairs on tibiae abundant and suberect; appressed pubescence sparse, tiny (up to $0.05 \mathrm{~mm}$ ) hairs.

Head and mesosoma coriaceous, gaster transversely striolate, all surfaces moderately to strongly shining. 


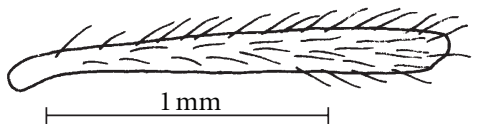

Figure 3: Posterior left tibia of a minor worker of C. reburrus (Napo, Ecuador).

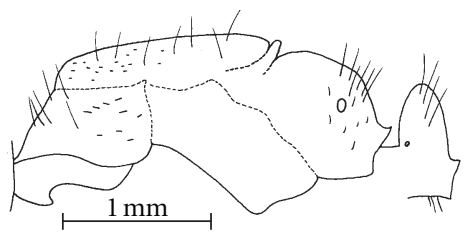

Figure 4: Mesosoma and petiole of the holotype female of C. reburrus.

Ferrugineous red, gaster with yellow lateral splotches ranging to striped brown and yellow.

3.2. Description of the Female. Female measurements $(\mathrm{mm}, n=4)$ : HL 1.66-1.74, HW 1.22-1.26, SL 1.32-1.40, EL 0.51-0.54, CL 0.56-0.58, CW 0.58, WL 2.62-2.68, FFL 1.44, FFW 0.38-0.39. Indices: CI 72-74, SI 79-81, CLI 100-102, FFI 26-27.

Clypeus with carina poorly developed; anterior clypeal border concave, medial portion meeting lateral portion in rounded angle; frontal carinae closely placed; eyes large, extending past sides of head; scape short, extending past posterior lateral corner of head by 1.5 funicular segments; head elongate, rectangular with nearly parallel sides; posterior margin convex, rounded; mesosoma elongate; pronotum lengthened and lowered in profile; metanotal suture deep, propodeum lower than remainder of mesosoma, rounded; petiole thick in profile.

Erect hairs abundant, about 20 scattered over surface of clypeus, 5-6 on cheek, 5 or more on malar area, erect hairs on posterior margin and posterior lateral corner of head, scape with two distinct types of hairs, erect (or nearly erect) longer $(0.13 \mathrm{~mm})$ hairs, and not very abundant (fewer than 10, excluding similar hairs at apex), and suberect short $(>0.08 \mathrm{~mm}$ ) abundant (about 100) hairs, pronotum with long $(0.4 \mathrm{~mm})$ erect hairs along posterior border, erect hairs scattered on dorsum of remainder of mesosoma, petiole and gaster, femora with several nearly erect hairs, tibiae with numerous hairs that are mostly suberect; appressed pubescence sparse, short on head $(0.02 \mathrm{~mm})$, longer on gaster $(0.06 \mathrm{~mm})$.

Sculpture finely coriaceous, but strongly shining.

Color predominantly dark brown, legs lighter brown, coxae, trochanters and base of tibiae yellow, gaster with lateral yellow blotches on each tergum.

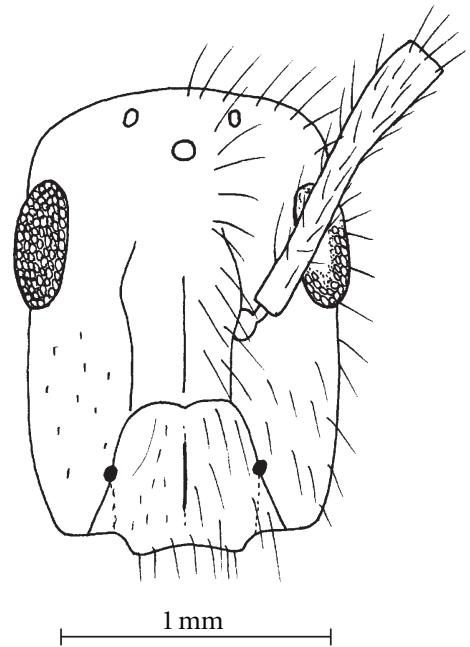

Figure 5: Head of the holotype female of C. reburrus.

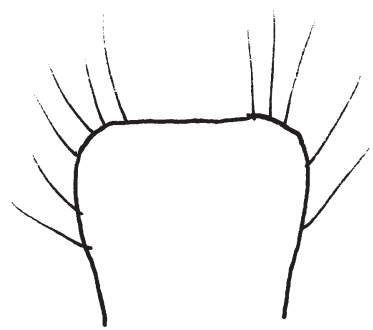

FIGURE 6: Petiole of the holotype female of C. reburrus, as seen from front.

FFW 0.24-0.25. Indices: CI 83-86, SI 92-94, CLI 117-121, FFI 18.

Mandible without teeth, except apical angle; anterior border of clypeus broadly convex, clypeal carina poorly defined, but present; sides of head anterior to eyes concave, nearly parallel, posterior border of head broadly rounded, concave between ocelli; scape extending approximately $1 / 2$ length past posterior lateral corner of head; eye large, occupying approximately 1/2 length of side of head; ocelli well developed; propodeal spiracle oval shaped; petiole thick when viewed in profile, apex slightly flat but convex as seen from front.

Erect and suberect hairs present on mandibles, clypeus, dorsum and ventral surfaces of head; sides of head, posterior border of head, dorsum of mesosoma, legs, petiole, and all surfaces of gaster; appressed hairs sparse few present on head, mesosoma, and gaster.

Dorsum of head coriaceous, mesosoma coriaceous but somewhat glossy, especially scutellum, side of pronotum, mesopleuron, gaster transversely striolate, moderately shining.

Pale medium brown, mandibles, cheeks, antennae, parts of scutum, legs yellowish brown.

Distribution. Known only from northeastern Ecuador. 


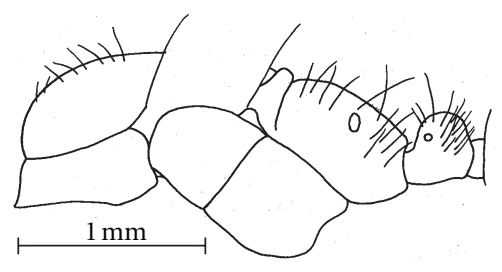

Figure 7: Mesosoma and petiole of a paratype male of C. reburrus.

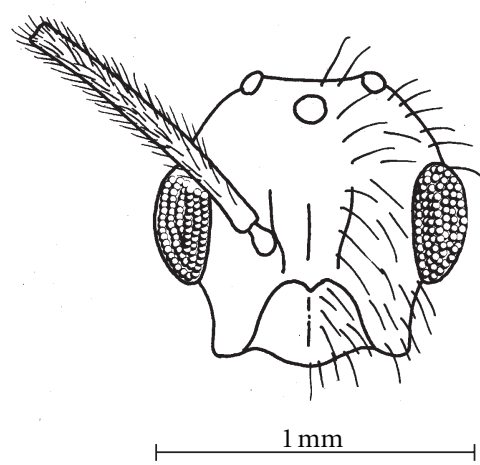

Figure 8: Head of a paratype male of C. reburrus.

Holotype Female. Ecuador, Napo, Sucumbíos, Sacha Lodge, $0.5^{\circ} \mathrm{S} 76.5^{\circ} \mathrm{W}, 290 \mathrm{~m}, 22$-ii-1994, P. Hibbs (Los Angeles County Museum of Natural History); Paratype females (3) same data as holotype, 4-iii-1994, 20,30-ix-1994, 10,21-xi1994 (Los Angeles County Museum of Natural History, Museo de Zoología, Pontificia Universidad Católica del Ecuador); additional paratypes including Orellana, Yasuni Research Station, $0^{\circ} 40^{\prime} \mathrm{S} 76^{\circ} 23^{\prime} \mathrm{W}, 200 \mathrm{~m}, 17-\mathrm{i}-2009$, C. Sagers and J. Trager numbers 2009-502, 2009-506, 2009510, 2009-515 (26 workers, 1 dealate female, 4 alate females, 4 males), same locality, 19-i-2009, P. Barriga and G. Alvia numbers 2009-021, 2009-028, 2009-030, 2009-031, 2009-037 (58 workers, 3 females, 18 males). The female was selected as the holotype as the minor workers, and males of Camponotus spp. are often indistinguishable. Paratypes will be distributed to major South American, North American, and European museums, including the California Academy of Sciences, Collection of William and Emma Mackay, University of Texas, El Paso, Ernst-Moritz-Arndt-Universitat, Greifswald, Germany, Los Angeles County Museum of Natural History, Museum of Comparative Zoology, Harvard University, Museo de Historia Natural, Universidad Nacional de Colombia, Museo de Zoología, Pontificia Universidad Católica del Ecuador, Museu de Zoología da Universidade de São Paulo, Brasil, Muséum d'Histore naturelle, Genéve, Switzerland and the Naturhistorisches Museum Wien, Burgring, Austria.

Material Examined. Type series and ECUADOR: Napo, $24 \mathrm{~km}$ NE Archidona, $0^{\circ} 43^{\prime} \mathrm{S} 77^{\circ} 41^{\prime} \mathrm{W}, 1000 \mathrm{~m}$, 7-viii-1991, P. Ward numbers 11394, 11396-2 (1 worker, 1 alate female, 1 dealate female, Museum of Comparative Zoology, Harvard University), $7 \mathrm{~km}$ ESE Puerto Misahualli, $1^{\circ} 4^{\prime} \mathrm{S} 77^{\circ} 37^{\prime} \mathrm{W}$, 400 m, 5-viii-1991, P. Ward numbers 11366 (3 workers John

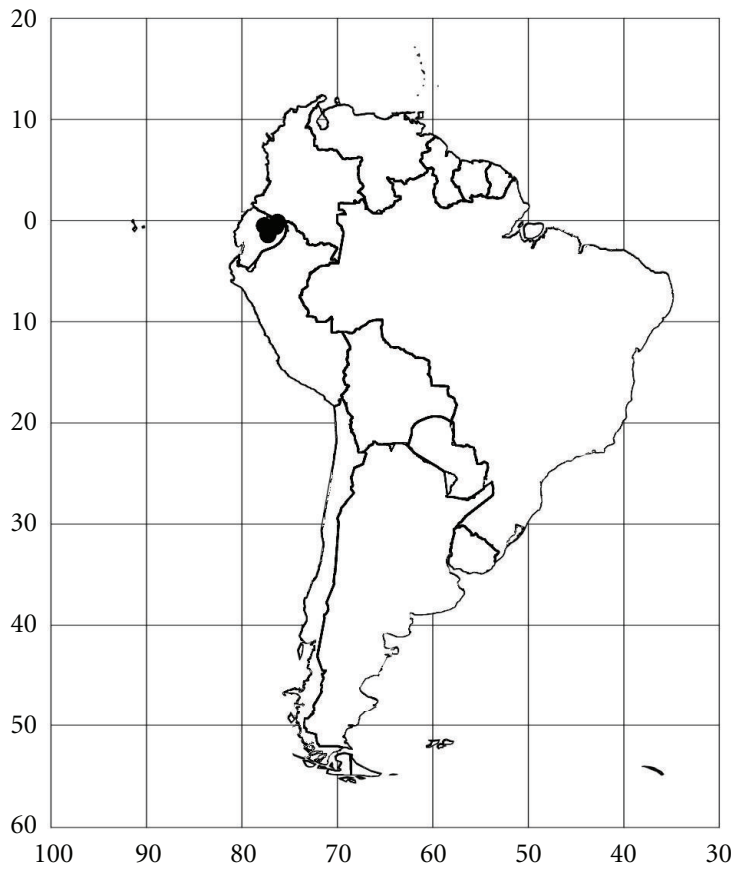

FIgURE 9: Camponotus reburrus.

Longino collection, 3 workers Museum of Comparative Zoology, Harvard University).

Etymology. From Latin, reburrus, meaning one with bristling hair, referring to the hairs on the head.

Comments. Camponotus reburrus does not appear to be closely related to any of the other described species in the subgenus Tanaemyrmex, other than C. balzani. This close relationship with C. balzani was further supported with DNA barcode analyses (Barriga, unpublished information).The minor workers can be separated in that the side of the head has few erect or suberect hairs (abundant in minors of C. balzani), the gaster is transversely striped with yellow bands (generally concolorous light brown in C. balzani, sometimes with a hint of tan transverse stripes), and the workers are slightly smaller (much overlap in size ranges in the two species). The females of $C$. reburrus are much smaller (maximum total length approaches $7 \mathrm{~mm}$ ) than those of $C$. balzani (total length over $1 \mathrm{~cm}$ ). The splotches or stripes on the gaster are definitely yellow in females of $C$. reburrus but are only pale brown (if present) on the gaster of females of C. balzani. The males of $C$. reburrus are also much smaller (total length approaching $4.7 \mathrm{~mm}$ ) as compared to the males of C. balzani (total length greater than $5 \mathrm{~mm}$ ). The males of C. reburrus are generally darker brown than the pale medium brown males of $C$. balzani. If major workers are found to exist, they will probably be similar to those of $C$. balzani with the sides of the head narrowed anteriorly, with a welldeveloped clypeal carina, but differing in having few erect hairs on the sides of the head. The gaster would be expected to have well-developed yellow splotches or bands, which 
would differ from the major of C. balzani in which the bands on the gaster are only pale brown, if present.

The abundant erect and suberect hairs on the scape and the abundant erect hairs on the clypeus suggest affiliation with species such as C. maculatus plombyi, but minor workers of $C$. reburrus differ in being much smaller and in that the head is little narrowed anteriorly as in C. plombyi. Camponotus reburrus could be confused with C. maculatus soulouquei, but it has little in common with this latter species. Camponotus reburrus can easily be separated by the shape of the head of the minor, which has parallel sides in C. reburrus and as the sides of the head of the minor is strongly narrowed anteriorly in C. maculatus soulouquei.

The yellow blotches on the gaster may result in $C$. reburrus being confused with members of the subgenus Myrmosphincta. Camponotus reburrus can be easily separated as the surface is nearly smooth (rough and punctate in Myrmosphincta) and the head is rectangular shaped (narrowed anteriorly in Myrmosphincta). Thus, C. reburrus would not be easily confused with any other South American species.

Biology. Three of the winged female-type specimens of $C$. reburrus were collected in a malaise trap. Others were collected in Cecropia at the edge of secondary growth rainforest or in gaps inside primary growth rainforest. Among the Cecropia species where this species was found are: C. marginalis Cuatrec, C. herthae Diels, and C. membranacea Trécul. The ants were found inside Cecropia internodes and were not aggressive as are Azteca ants. Müllerian bodies and scale insects were found in the internodes where the ant species were living. This species is smaller than C. balzani, but the behavior inside Cecropia nodes is similar: the ants of both species do not attack but run and escape. Camponotus balzani and C. reburrus inhabit Cecropia juvenile and reproductive plants (plants with pistillate or staminate flowers), but most Cecropia species were found more commonly with aggressive colonies of Azteca ants.

\section{Acknowledgments}

The authors thank the Ernst Mayr fund of the Museum of Comparative Zoology of Harvard University for funding several trips to compare type material and to see the extensive collection of C. balzani. Gary Alpert provided lodging, and Stefan Cover allowed access to the ant collection. Gary Alpert will also put colored photographs of paratypes on the ant species website of the Museum of Comparative Zoology http://insects.oeb.harvard.edu/mcz/. The Ministerio del Ambiente of Ecuador, the World Wildlife Fund's Russell E. Train Education for Nature Program and the National Science Foundation, Office of International Science and Education Grant no. 0813594 provided funding for the project. They would like to thank an anonymous reviewer for correcting several mistakes and making important suggestions that improved the paper.

\section{References}

[1] E. O. Wilson, Pheidole in the New World, a Domin Ant, Hyperdiverse Ant Genus, Harvard University Press, 2003.

[2] W. Mackay and T. Delsinne, "A new species of carpenter ant (Hymenoptera: Formicidae: Camponotus) from Paraguay with a key to the new world members of the maculatus species complex," Sociobiology, vol. 53, no. 2, pp. 487-498, 2009.

[3] W. P. Mackay, C. López-Castro, and F. Fernández, "A new, high altitude Colombian species of the ant genus Camponotus with dimorphic males and females (Hymenoptera: Formicidae)," Sociobiology, vol. 40, no. 2, pp. 421-430, 2002.

[4] W. P. Mackay, M. A. Mackay, and E. E. Mackay, "An unusual new carpenter ant of the genus Camponotus (Hymenoptera: Formicidae) from Nicaragua," Sociobiology, vol. 43, no. 3, pp. 459-465, 2004.

[5] W. P. Mackay and E. E. Mackay, "A revision of the neotropical ants of the montivagus species complex, genus Camponotus, subgenus Myrmentoma (Hymenoptera: Formicidae)," Sociobiology, vol. 30, no. 3, pp. 319-334, 1997.

[6] R. R. Snelling, "A review of the Camponotus montivagus complex (Hymenoptera: Formicidae)," Sociobiology, vol. 36, no. 3, pp. 599-611, 2000.

[7] F. Fernández, "Revisión de las hormigas Camponotus subgénero Dendromyrmex," Papéis Avulsos de Zoologia, vol. 42, pp. 47-101, 2002. 

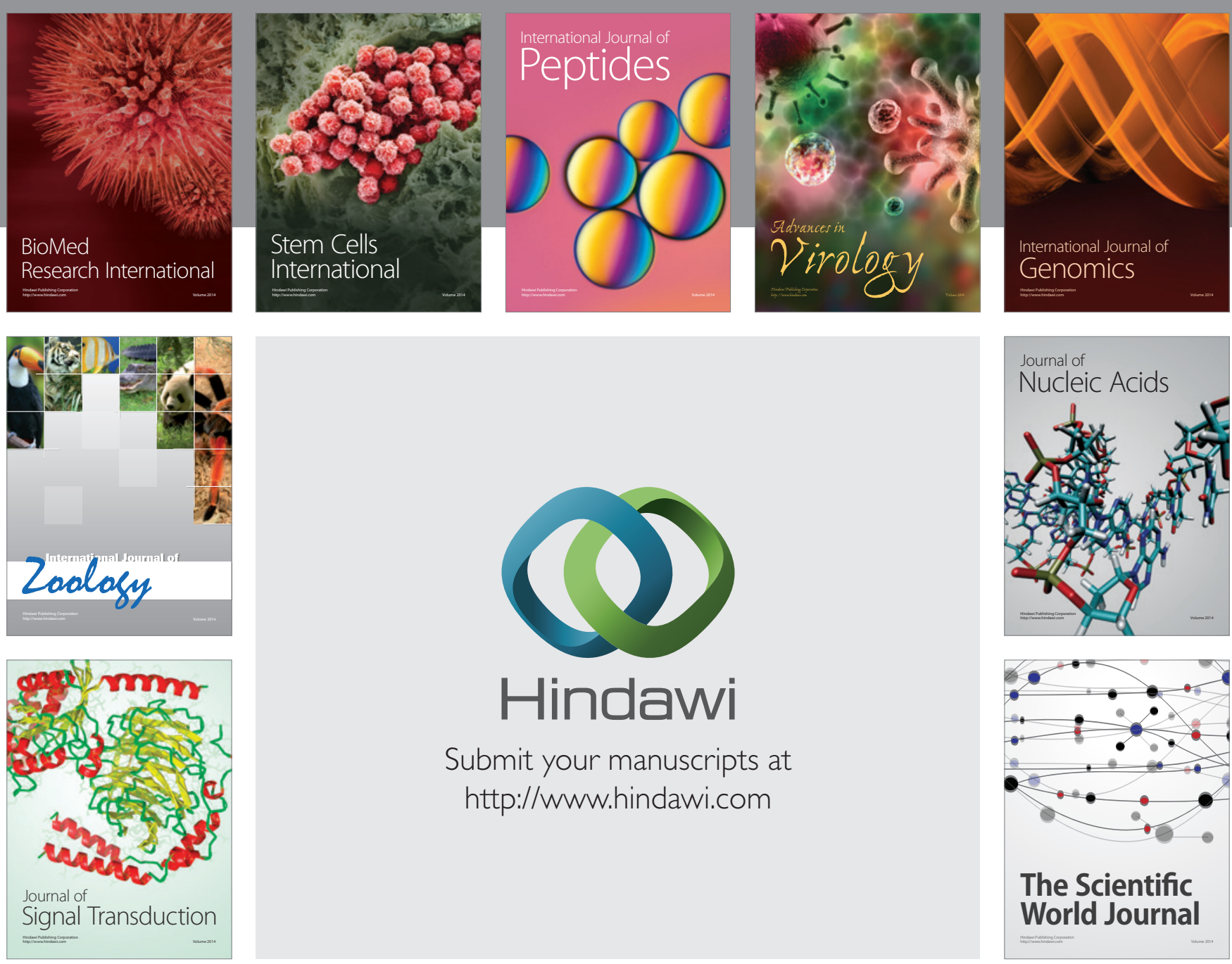

Submit your manuscripts at

http://www.hindawi.com
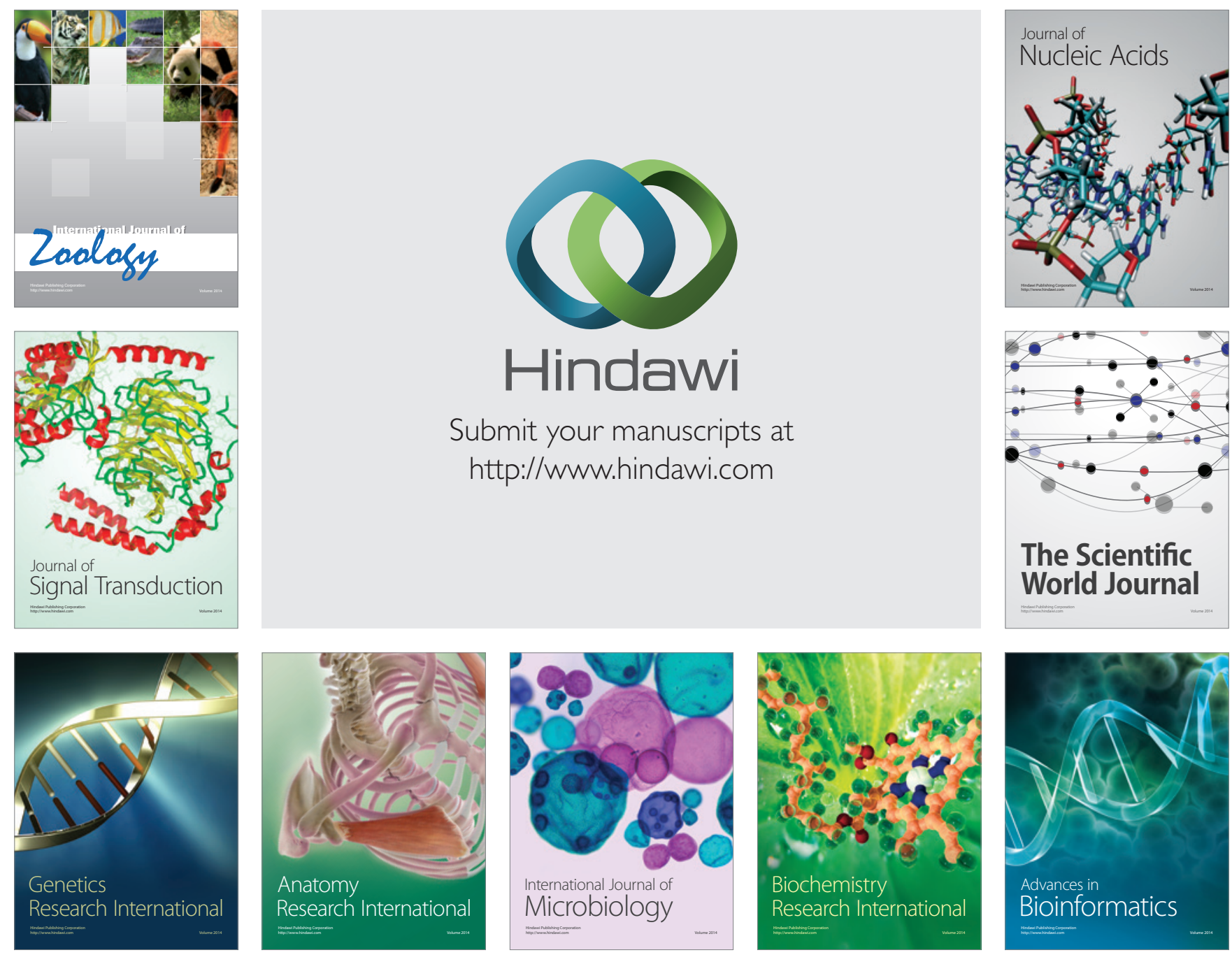

The Scientific World Journal
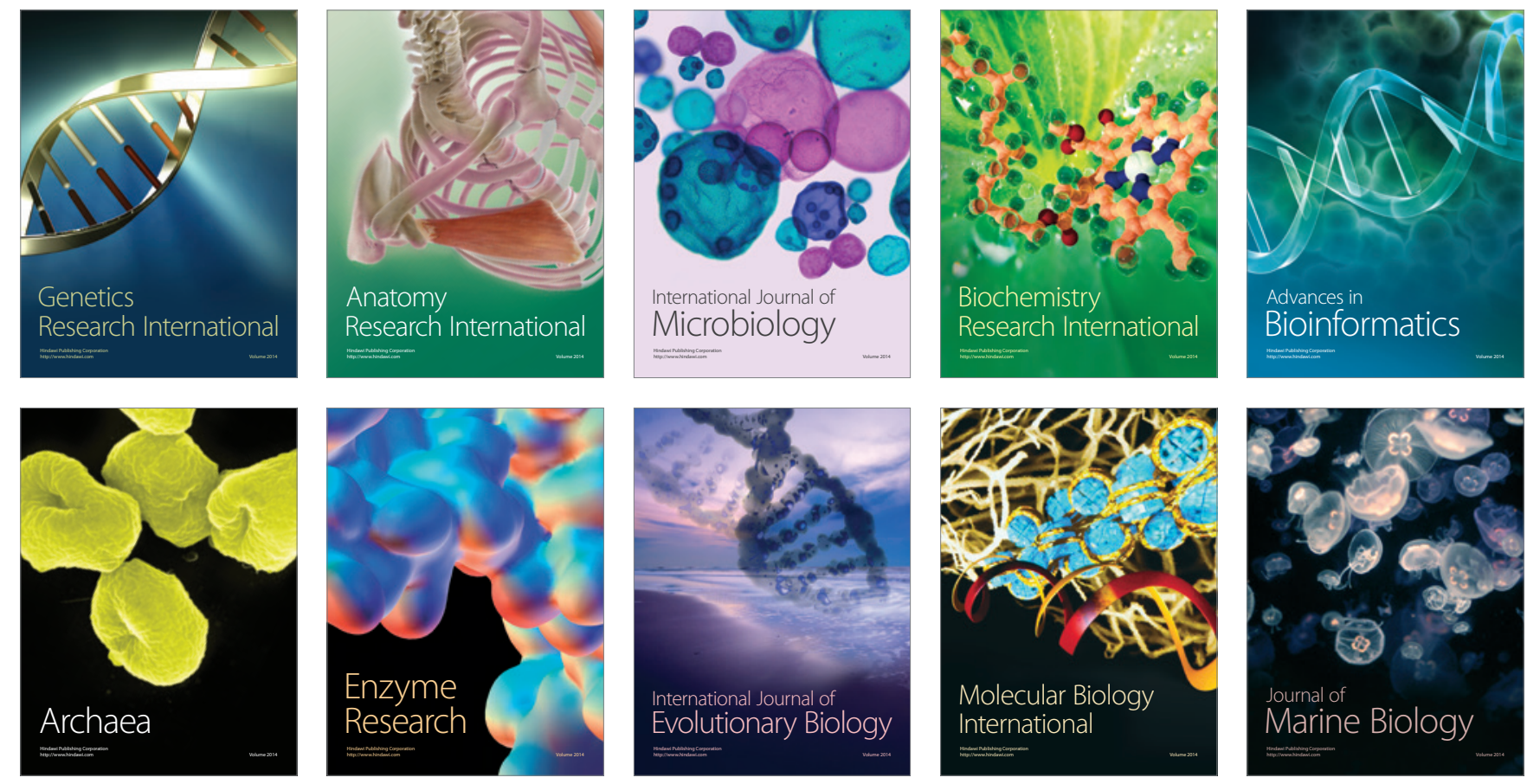